Ana Lucia Falavigna-Guilherme 1

Arnaldo Lima Costa 2

Osmar Batista 2

Gilberto Cezar Pavanelli 1

Silvana Marques de Araújo 3

\section{Atividades educativas para o controle de triatomíneos em área de vigilância epidemiológica do Estado do Paraná, Brasil}

\author{
Health education for triatomine control \\ in an area under epidemiological surveillance \\ in Paraná State, Brazil
}

1 Curso de Pós-graduação em Ecologia de Ambientes Aquáticos Continentais, Centro de Ciências Biológicas, Universidade Estadual de Maringá. Av. Colombo 5790, Maringá, $P R$ 87020-900, Brasil. alf@wnet.com.br

2 Fundação Nacional de Saúde, Subdistrito de Maringá. Av. Dr. Gastão Vidigal 55, Maringá, $P R$

87050-440, Brasil.

3 Departamento de Análises Clínicas, Setor de Parasitologia Básica, Universidade Estadual de Maringá.

Av. Colombo 5790 , Maringá, $P R$ 87020-900, Brasil.

\begin{abstract}
This paper describes a health education project targeting a rural community and health professionals from counties undergoing epidemiological surveillance for Chagas disease vectors in the State of Paraná, Brazil. A group of technicians from the Brazilian National Health Foundation (FUNASA) was trained, together with teachers, workers, and students from the State University of Maringá, who prepared an instructions manual and drew up guidelines for reporting presence of triatomines or suspected cases of Trypanosoma cruzi infection. From June 1996 through February 2000, the activities reached 742 families, 2,300 schoolchildren, and 27 teachers from 18 elementary schools, and included a meeting between FUNASA members and 40 participants, 21 meetings in health centers, and provision of a set of preserved triatomine specimens for use in vector surveillance and identification. After three years of health education activities and insecticide treatment (cipermetrina $125 \mathrm{mg}$ i.a. $/ \mathrm{m}^{2}$ ), there was a reduction of $80.6 \%$ in households infested with triatomines and increased awareness among rural residents and health workers. The authors discuss the need to train professionals committed to changing Brazil's prevailing health model.
\end{abstract}

Key words Health Education; Triatominae; Chagas Disease; Epidemiologic Surveillance

Resumo O objetivo deste artigo é descrever um projeto de atividades de educação sanitária para a comunidade rural e profissionais de saúde de municípios em fase de vigilância epidemiológica de triatomíneos no Paraná. Uma equipe composta de técnicos da Fundação Nacional de Saúde, professores, funcionários e acadêmicos da Universidade Estadual de Maringá foi treinada, preparando um manual de orientações e elaborando um fluxo a ser seguido em caso de denúncia de triatomíneo ou de indivíduo com suspeita de infecção pelo Trypanosoma cruzi. De junho de 1996 a fevereiro de 2000, as atividades incluíram 742 famílias, 2.300 alunos e 27 professores em 18 escolas de ensino fundamental, incluindo um evento para especialistas com 40 participantes, 21 reuniões em Postos de Saúde e a entrega de um mostruário com triatomíneos. Três anos após a realização dessas atividades e aspersão de inseticida (cipermetrina $125 \mathrm{mg}$ i.a./m²), verificou-se redução de $80,6 \%$ das unidades domiciliares infestadas por triatomíneos, além da conscientização da comunidade rural e profissionais de saúde. Foi discutida a necessidade de formação de profissionais compromissados com a inversão do modelo de saúde vigente no País. Palavras-chave Educação em Saúde; Triatominae; Doença de Chagas; Vigilância Epidemiológica 


\section{Introdução}

O impacto ocorrido sobre a transmissão da doença de Chagas no Brasil foi evidente em virtude das ações de controle centradas no tratamento químico domiciliar, principalmente entre os anos de 1975 a 1983 (Silveira \& Vinhaes, 1998). Além do uso de inseticidas, a vigilância entomológica deve ser de caráter permanente, exercida pela população e pelos serviços de saúde, para dar sustentabilidade ao controle alcançado (Silveira \& Vinhaes, 1998). Segundo Avila et al. (1998), a educação em saúde deverá sempre ser incluída como um componente dos programas de controle, e a importância dos triatomíneos como transmissores deverá ser enfatizada, tanto quanto a melhoria dos domicílios, com a participação da comunidade como uma medida efetiva e sustentável.

O processo de detecção de triatomíneos com a participação da população significa uma vigilância contínua, se comparado às atividades rotineiras realizadas pelas equipes de profissionais de campo da Fundação Nacional de Saúde - FUNASA (Silva et al., 1999). As ações dirigidas aos indivíduos visando à promoção de saúde devem estar voltadas aos seus hábitos, comportamentos e práticas sociais (Mello et al., 1992).

Em artigos recentes para pesquisa de triatomíneos na área rural de municípios do noroeste do Estado do Paraná (Falavigna-Guilherme et al., 2001, submetido), observou-se a presença de Triatoma sordida e Panstrongylus megistus no peridomicílio e até mesmo em alguns domicílios. Durante as pesquisas, estes autores verificaram um grande desinteresse por parte dos moradores com relação a esses achados. Alguns moradores revelaram ainda desconhecer as conseqüências do fato, alegando ser esse “... um problema da SUCAM”. Da mesma forma, verificaram que os responsáveis pela saúde nos municípios incluídos no estudo quase sempre não orientavam adequadamente, em resposta às solicitações da comunidade sobre como e a quem encaminhar denúncias de triatomíneos e pacientes chagásicos. Assim, o presente trabalho teve como objetivo promover atividades de educação sanitária voltadas ao controle de triatomíneos para a comunidade rural e para profissionais de saúde dos municípios investigados por Falavigna-Guilherme et al. (2001, submetido).

\section{Material e métodos}

\section{Descrição da região em estudo}

O norte e noroeste do Paraná compunham, até por volta de 1970, uma das regiões mais bem estruturada do País em termos da malha fundiária calcada na pequena e média propriedade e no uso da terra (Alegre \& Moro, 1986). Na colonização, a área predominantemente cafeeira abrigava numerosa população, principalmente de paulistas e mineiros, que em pouco tempo se despovoou (Alegre \& Moro, 1986).

Os dados referentes ao encontro de triatomíneos no Estado do Paraná estão desatualizados (Almeida, 1948; Araújo, 1954; Lobo et al., 1952; Silveira et al., 1984; Simões, 1943). Segundo a FUNASA/Paraná, o último relato de encontro da espécie Triatoma infestans na região noroeste do Paraná foi em 1986. Silveira et al. (1984) relataram o encontro freqüente também das espécies T. sordida e P. megistus. Mais recentemente, Toledo et al. (1997) verificaram que $T$. sordida correspondeu a $98,7 \%$ dos triatomíneos coletados em domicílios e anexos no peridomicílio, na zona rural de municípios do noroeste do Paraná. Todavia, Silveira \& Vinhaes (1998) não relataram o encontro de $P$. megistus e T. sordida neste Estado de 1995 a 1997.

Camargo et al. (1984) verificaram que o Estado do Paraná teve 4,0\% da população rural com sorologia positiva para doença de Chagas. Com base nesta prevalência, Silveira \& Resende (1994) estimaram em 166.511 o número de casos positivos para este Estado com população rural de 4.164.943 habitantes.

Mais recentemente, Falavigna-Guilherme et al. (2001, submetido) verificaram que, de 567 unidades domiciliares rurais (UDS) pesquisadas no noroeste do Estado do Paraná, 72 (12,7\%) apresentavam-se infestadas, sendo 56/509 (11,1\%) habitadas e 16/58 (27,6\%) desabitadas. Foram capturados 329 ninfas e 123 adultos de T. sordi$d a$; 41 ninfas e 28 adultos de $P$. megistus e um adulto de Rhodnius neglectus, sendo os exemplares destes insetos capturados prevalentemente no peridomicílio.

Conforme mostra a Figura 1, foram trabalhados os municípios de Paiçandu, Dr. Camargo, Floresta, Marialva, Mandaguari, Ourizona, Porto Rico, Maringá e o Distrito de Floriano, localizados no noroeste do Estado, por serem municípios onde freqüentemente ocorriam denúncias do encontro de triatomíneos; Cândido de Abreu, localizado no centro do Estado do Paraná, por ser considerado de prioridade I pela FUNASA, ou seja, com possibilidade de se encontrar T. infestans. As unidades domiciliares 
infestadas de cada município trabalhado foram desinsetizadas com cipermetrina $125 \mathrm{mg}$ i.a. $/ \mathrm{m}^{2}$. O trabalho foi realizado de junho de 1996 a fevereiro de 2000.

Com relação à doença de Chagas, não havia um trabalho articulado entre SUS e FUNASA, e esta se restringia ao atendimento dos casos de denúncias do encontro de triatomíneos.

\section{Preparação da equipe técnica}

Para fazer a educação sanitária individualmente a cada família visitada, foram preparados oito alunos bolsistas do Programa Institucional de Bolsas de Iniciação Científica (PIBIC), dois técnicos de nível médio, três auxiliares de laboratório e dois docentes da Universidade Estadual de Maringá (UEM).

Para o trabalho educativo com os escolares do ensino fundamental, foram preparados 80 acadêmicos dos cursos de Farmácia e Enfermagem por meio de palestras, discussões e grupos de estudo dirigido. Essas atividades constaram do programa e da avaliação dos acadêmicos nos dois cursos supracitados.

Foi realizado um curso teórico-prático de 40 horas para 42 agentes da FUNASA que também participaram da equipe de educação sanitária.

Cada categoria profissional contribuiu com o seu conhecimento para a formação de um texto único que serviu para a padronização das informações a serem transmitidas à populaçãoalvo, o que foi feito com treinamento priorizando esclarecimentos sobre controle de triatomíneos e sua importância. Com base nos achados de Falavigna-Guilherme et al. (2001, submetido), a FUNASA juntamente com a UEM, sem consulta prévia à população, decidiram realizar campanhas educativas sobre educação em saúde enfocando o controle da doença de Chagas.

Foi também apresentado um fluxo a ser seguido em caso de denúncia de triatomíneo ou de indivíduo com suspeita de infecção pelo Trypanosoma cruzi. No caso de denúncia, o morador deveria dirigir-se ao posto de saúde mais próximo ou à Secretaria de Saúde Municipal. Estes órgãos deveriam recorrer à FUNASA ou à universidade, as quais se comprometeriam em retornar as informações ou soluções solicitadas. O fluxo de atendimento ao paciente com diagnóstico positivo ou com suspeita clínica consistiria no seu encaminhamento ao Laboratório de Doença de Chagas da UEM ou ao Programa de Atenção ao Chagásico com Educação Integral (ACHEI) (Araújo et al., 2000).

O treinamento reforçava que essas informações deveriam ser transmitidas em todas as instâncias, e o tipo de atividade e o aprofunda-
Figura 1

Municípios do Estado do Paraná, Brasil, em que foram realizadas atividades educativas para o controle de triatomíneos. Junho de 1996 a fevereiro de 2000

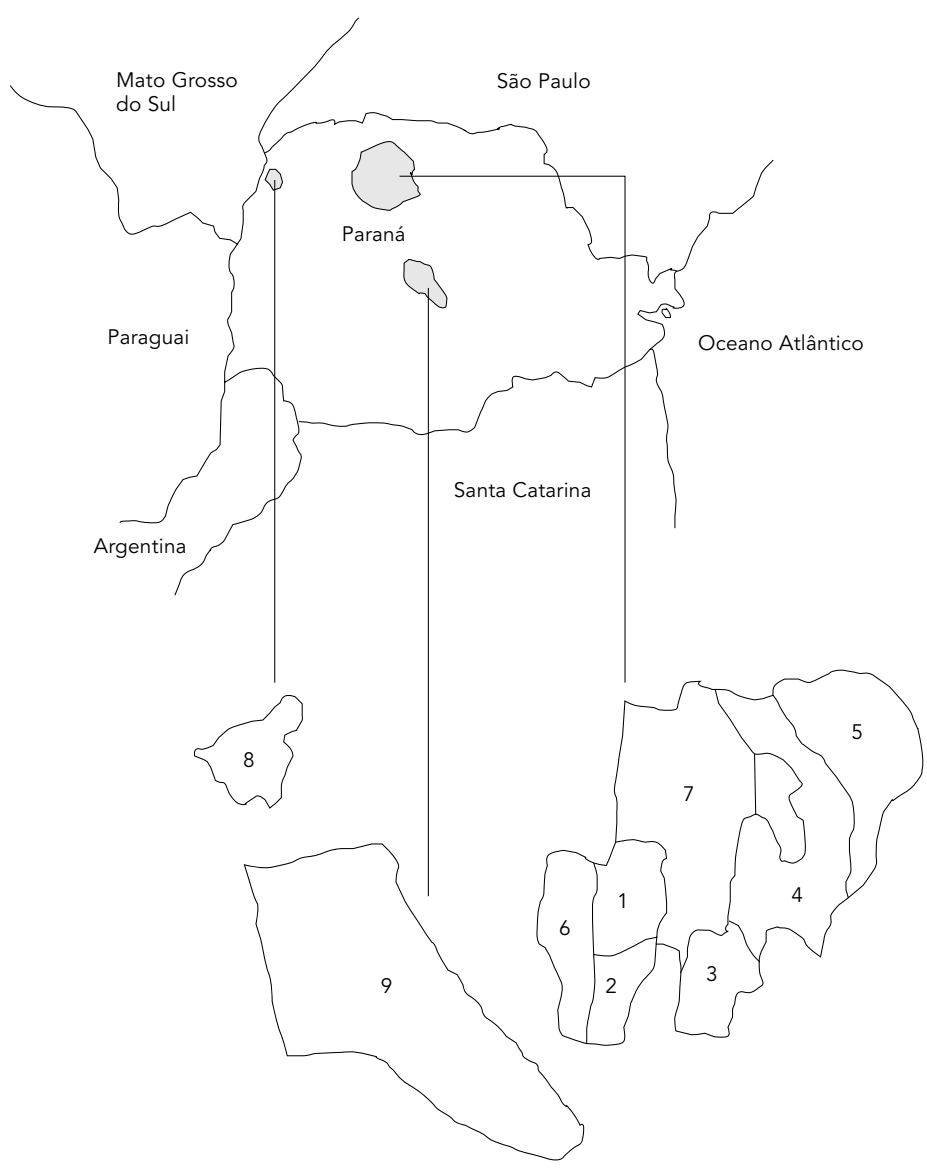

$$
\begin{aligned}
& 1 \text { - Paiçandu } \\
& 2 \text { - Dr. Camargo } \\
& \text { 3 - Floresta } \\
& \text { 4 - Marialva } \\
& 5 \text { - Mandaguari } \\
& \text { 6- Ourizona } \\
& 7 \text { - Maringá } \\
& \text { 8 - Porto Rico } \\
& 9 \text { - Cândido de Abreu }
\end{aligned}
$$

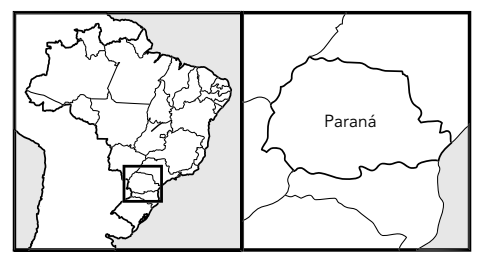

mento do conteúdo deveriam ser escolhidos de acordo com o público em questão. Foram ainda convidados pesquisadores renomados em assuntos específicos da doença de Chagas para atualização de profissionais especialistas.

Foi confeccionado um manual para orientação de escolares e moradores rurais, com ilustrações didáticas e informações básicas e esclarecedoras, como os exemplos a seguir: "O que devemos fazer para combater o barbeiro? Melhorar a nossa casa, tapando os buracos da pa- 
rede, do piso, não deixando lenha amontoada dentro de casa ou no quintal, para que o barbeiro não encontre lugar para se esconder" (Falavigna-Guilherme et al., 1999:1).

"O que a pessoa que tem Chagas pode sentir? No início a doença pode nem ser percebida. Muitos anos mais tarde a pessoa pode sentir palpitação, falta de ar, canseira..." (FalavignaGuilherme et al., 1999:6).

Especificamente esse manual teve como base um informativo da FUNASA. Abordava de forma lúdica e rápida o habitat dos triatomíneos na mata e no domicílio, o que deve ser feito para combater estes insetos e o que aconteceria se porventura o homem fosse sugado por um barbeiro infectado; dava também alguns esclarecimentos sobre sintomas da doença de Chagas. Apresentava ainda a FUNASA e o posto de saúde mais próximo como referenciais para maiores informações.

\section{População alvo}

\section{- Moradores da área rural}

As investigações de Falavigna-Guilherme et al. (2001, submetido) realizaram-se uma vez por semana por técnicos da UEM e da FUNASA. Durante esse período, enquanto era feita a investigação do domicílio e seus anexos para pesquisa de triatomíneos, um componente da equipe de educação sanitária permanecia junto ao morador a fim de prestar esclarecimentos básicos de higiene e saúde, enfocando limpeza do domicílio, peridomicílio e medidas de controle de triatomíneos. Durante a explanação, eram mostrados aos indivíduos exemplares de ninfas e adultos de T. sordida e P. megistus. Para as localidades onde as propriedades rurais apresentaram-se mais infestadas por triatomíneos, realizaram-se ainda reuniões coletivas de esclarecimento. Nestas reuniões utilizavam-se vocabulário adequado, recursos audiovisuais, panfletos e exemplares de triatomíneos. Ao fim de cada palestra, era feito um debate para que os moradores observassem as melhorias que eles próprios poderiam realizar em suas propriedades, além de solucionar as dúvidas levantadas. A comunidade era também orientada com relação ao que fazer e a quem procurar no caso de encontrar triatomíneos. O manual de orientação foi entregue a cada família.

\section{- Escolares e professores do ensino fundamental}

Foram incluídos no trabalho alunos e professores de escolas rurais ou urbanas que recebes- sem maior número de alunos do meio rural. Elegeram-se os municípios de Paiçandu, Floresta, Doutor Camargo, Maringá e o Distrito de Floriano, por apresentarem maior infestação por triatomíneos. O conteúdo foi apresentado na forma de atividades lúdicas, como teatros, fantoches, brincadeiras e palestras com recursos audiovisuais. Além de se distribuir a cada escolar o manual de orientações, a escola e seus professores receberam outros informativos técnicos para que servissem de apoio em outros trabalhos a serem realizados em sala de aula.

\section{- Profissionais de saúde}

Foram incluídos funcionários dos serviços de vigilância entomológica dos municípios, agentes comunitários, enfermeiras, atendentes, secretários de saúde e representantes de pastorais da saúde. Foram contemplados todos os municípios, dando maior atenção àqueles de maior infestação de triatomíneos. Para homogeneização do conhecimento específico, promoveram-se reuniões educativas com informações atualizadas, priorizando-se para os profissionais a importância do acolhimento de solicitações e dúvidas da comunidade com relação a insetos suspeitos, seu encaminhamento ao órgão de saúde adequado e o retorno da informação à população.

\section{- Profissionais de bancos de sangue e médicos de especialidades}

A estes profissionais, foi apresentado o fluxo de atendimento ao paciente chagásico criado pelo Laboratório de Doença de Chagas da UEM, a situação atual da infestação por espécies secundárias de triatomíneos no Paraná e conhecimentos atuais sobre o tratamento específico para doença de Chagas.

\section{Avaliação do trabalho}

A avaliação das atividades educativas foi realizada ao longo do projeto, durante cada atividade, avaliando-se também a percepção do aumento das demandas de encaminhamentos de triatomíneos e de pacientes para a FUNASA/ UEM. Três anos após o trabalho realizado por Falavigna-Guilherme et al. (2001, submetido), no Município de Paiçandu, que apresentou maior grau de infestação triatomínica, foi feita nova avaliação dessa infestação nas UDS rurais para verificar o impacto da aspersão de inseticida e das atividades de educação sanitária. Resumidamente, a inspeção entomológica, desenvolvida segundo descrito anteriormente 
por Falavigna-Guilherme et al. (2001), foi feita por uma equipe de seis agentes de saúde da FUNASA, treinada para busca entomológica e aspersão de inseticida. Os triatomíneos foram capturados manualmente nas paredes externas, internas e nas coberturas das casas habitadas ou desabitadas, em móveis, colchões e outros objetos. Procedimento similar foi adotado em relação aos anexos. Foram consideradas positivas as UDS onde foram encontradas ninfas e/ou adultos de triatomíneos. As UDS infestadas eram borrifadas com cipermetrina (125mg i.a. $/ \mathrm{m}^{2}$ ), após a inspeção entomológica.

\section{Resultados}

\section{Atividades direcionadas}

a moradores da área rural

Foram trabalhadas individualmente 742 famílias. Três reuniões foram realizadas em três localidades distintas do Município de Paiçandu, onde foi verificada elevada infestação por triatomíneos. Essas reuniões tiveram participação média de 35 pessoas em cada uma delas, oportunidades em que foi constatado que moradores mais antigos preservavam a informação de como é o "barbeiro" e quais os riscos que este pode causar ao homem. Outro dado constatado foi o descaso com o peridomicílio que, independente das condições de cuidado do domicílio, era deixado em abandono. É freqüente a existência de pequenos proprietários rurais que residem no meio urbano e que mantém o peridomicílio de suas casas organizado de forma semelhante ao existente no meio rural. Foi verificado que era difícil para a população limpar todo o peridomicílio porque percebia que o mesmo não fazia parte de sua moradia. A sensação era de que a proximidade da área rural com o centro urbano gerava em seus moradores comportamento semelhante ao do morador urbano, que vai do trabalho a casa no final do dia, confinando-se no domicílio esquecendo tudo que está ao seu redor. A população moderna, de modo geral, desconhece o triatomíneo, e as casas abandonadas no peridomicílio ou em suas proximidades podem ser focos de infestação porque geralmente, na região, os moradores as utilizam como depósitos de madeira.

Foi também percebido pela fala dos moradores que "bicho barbeiro já existiu na região $e$ hoje não existe mais; nossa mais ainda esta estória de barbeiro...; que ser picado pelo barbeiro é coisa do passado" e que "a culpa da moradia infestada é dos órgãos de saúde”. Esta concepção foi identificada tanto entre os moradores rurais e professores do ensino fundamental, quanto entre os profissionais dos órgãos de saúde, deixando clara a necessidade de promover formas efetivas de atualização destes profissionais.

No Centro Comunitário do Distrito de Água Boa, Paiçandu, promoveu-se uma Feira de Controle de Vetores - Triatomíneos e Aedes. Participaram do evento 300 pessoas. Foram ainda realizadas em Paiçandu duas reuniões na Secretaria de Saúde com a participação de vinte líderes comunitários.

Em todas essas oportunidades foi sugerida a reorganização do peridomicílio, priorizando sempre a sua limpeza, queima de entulhos, uso periódico de inseticidas e pesticidas, e qualquer inseto suspeito deveria ser encaminhado rapidamente ao posto de saúde mais próximo. Foi também enfatizado que, na prevenção de doenças, cada um tem uma parcela de responsabilidade, e o cuidado com a moradia compete aos moradores.

Em 1996, o trabalho de campo realizado no Município de Paiçandu investigou 72 UDS e 31 $(43,1 \%)$ estavam infestadas por triatomíneos. Em 1999, após a aspersão de inseticida e as atividades educativas, verificou-se redução de $80,6 \%$ de infestação das UDS, sendo constatado grande empenho dos moradores, principalmente no que diz respeito à limpeza e melhoria do peridomicílio.

\section{Atividades direcionadas a escolares e professores do ensino fundamental}

Foram realizadas atividades educativas em 18 escolas, com a participação de 2.300 alunos da primeira à oitava série. $\mathrm{O}$ aproveitamento foi verificado ao final de cada atividade, quando a platéia era convidada a responder questões sobre os principais aspectos abordados. As respostas certas foram premiadas, o que garantiu a participação e interesse geral dos escolares. O manual de orientações foi entregue com a tarefa de que cada aluno discutisse o conteúdo com seus pais. Como fruto dessa atividade, professores de algumas escolas relataram que foram procurados por pais para obter maiores informações sobre o assunto. De uma maneira geral, essas preocupações relacionavam-se com o temor da volta da doença transmitida pelo "bicho barbeiro".

Foi realizada, ainda, uma palestra que contou com a presença de 27 professores da rede pública dos municípios de Floresta e Paiçandu. 
Atividades direcionadas

a profissionais de saúde

Foram realizadas 21 reuniões em Postos de Saúde e Secretarias Municipais de Saúde dos Municípios de Paiçandu, Floresta, Floriano, Mandaguari, Marialva e Maringá. As platéias congregaram do atendente de balcão do Posto de Saúde ao secretário de saúde do município. Durante as discussões que se seguiram a estas reuniões, foi observado que estes profissionais não tinham idéia do procedimento correto a ser adotado em face de denúncias de triatomíneos ou de insetos remetidos a estes órgãos. Algumas vezes, o profissional devolvia o inseto ao morador dizendo não saber qual o encaminhamento a ser dado ao mesmo.

Cada Posto de Saúde ou Secretaria de Saúde recebeu um mostruário com ninfas e adultos de $T$ sordida e $P$. megistus, o manual de orientações e panfletos informativos do programa ACHEI.

Os postos de saúde da região em estudo passaram a usufruir os serviços oferecidos pela FUNASA e UEM para esclarecimentos de dúvidas e busca de soluções. Mais importante é que esses postos passaram a encaminhar as denúncias de moradores para a FUNASA.

\section{Atividades direcionadas a médicos \\ de especialidades e profissionais \\ de bancos de sangue}

Foi realizado um evento comemorativo dos 90 anos da descoberta da doença de Chagas no Hemocentro do Hospital Universitário da Universidade Estadual de Maringá, Paraná, em que se contou com a presença de palestrantes renomados de São Paulo e Minas Gerais. Participaram do evento 40 profissionais e lideranças da FUNASA/Paraná, e, além do assunto proposto, foi oportuna a discussão levantada sobre a municipalização da FUNASA.

Esse evento proporcionou o estreitamento das relações entre universidade, bancos de sangue, profissionais especialistas e FUNASA. Essa proximidade entre os diversos serviços trouxe vantagens para os profissionais e para os pacientes atendidos, já que não só os profissionais se beneficiam com a troca de informações técnicas, como também os pacientes recebem resultados mais seguros graças ao fluxo de checagem dos resultados, criado entre universidade e bancos de sangue. Também se observou maior número de encaminhamentos de indivíduos positivos para T. cruzi oriundos de bancos de sangue e postos de saúde ao programa ACHEI.

\section{Discussão}

Neste trabalho, foram relatadas diferentes atividades educativas direcionadas a moradores da zona rural, professores do ensino fundamental e profissionais de saúde, a fim de contribuir para as ações de controle da doença de Chagas no Paraná. Os programas conduzidos pelo Ministério da Saúde brasileiro que visam consolidar o controle dessa endemia passam pelas ações de educação da população (Willians-Banglero et al., 1999). Além disso, a inversão do modelo assistencial vigente no país, de curativo para preventivo, inclui formação acadêmica diferenciada, conforme discutido na Reunião Técnicas dos Pólos de Capacitação, Formação e educação Permanente em Saúde da Família, em 1999.

Nessa concepção, este trabalho envolveu acadêmicos em atividades extramuros, para que os mesmos vivenciassem problemas de saúde da comunidade e participassem das soluções propostas.

As atividades direcionadas à comunidade rural buscaram dar-lhe informação e envolvêla, no sentido de ser um parceiro na vigilância entomológica, solicitando sempre que possível a presença do morador no decorrer da investigação triatomínica, orientando-o quanto aos locais mais comuns do encontro dos insetos transmissores da doença. Embora não tenha havido supervisão e controle de vigilância efetivo, observou-se, três anos mais tarde, no Município de Paiçandu, redução no índice de infestação triatomínica. Ficou claro que isso se deveu a uma ação integrada envolvendo as atividades educativas e aspersão de inseticida pela FUNASA, prontamente aceitas pela comunidade. Sabe-se que apenas a utilização do inseticida sem a participação dos moradores não seria suficiente para manter as UDS livres da infestação, lembrando que o peridomicílio foi o local mais infestado e que a participação da comunidade foi efetiva dando maior atenção à limpeza deste. O inseticida usado em anexos mais expostos às adversidades do meio ambiente com maior grau de exposição solar, ventos e chuvas, pode ter seu tempo de ação reduzido (Nascimento et al., 1997).

Dias \& Garcia (1978), em Bambuí, Minas Gerais, demonstraram que a população responde de forma efetiva às ações de seus interesses na medida em que compreende os processos e os problemas que lhe afetam de modo direto. A literatura relata que o envolvimento da população em ações de vigilância vetorial da doença de Chagas foi mais eficaz e menos dispendiosa que as intervenções verticais tanto no Brasil, como em outros países (Silva et al., 1999). 
O enfoque dado aos cuidados com o peridomicílio vem ao encontro dos achados de Dias (1999). Este autor afirmou que, com a drástica redução da infestação domiciliar, os triatomíneos encontrados na atualidade constituem resquícios peridomiciliares, onde os inseticidas não funcionam bem, sendo imprescindível a participação dos moradores no que toca à organização, à limpeza periódica e ao manejo deste espaço (Dias, 1999).

A inclusão de líderes comunitários nas atividades do trabalho foi importante para garantir continuidade das ações. Garcia-Zapata \& Marsden (1993), num programa de controle da doença de Chagas em Goiás, contaram com esse grupo de voluntários para prestar atendimento ao morador do campo, por meio de atividades educativas, coleta de triatomíneos e trabalhos de desinsetização.

O escolar foi um dos alvos do presente trabalho. Segundo Hollanda (1992), os hábitos da população são dificilmente mudados, mesmo quando se trabalha intensamente com uma equipe de educação sanitária. Entretanto, parte dessas dificuldades pode ser contornada pelo envolvimento de crianças em atividades que têm como fim mudanças comportamentais, por serem elas mais receptivas, podendo funcionar como agentes multiplicadores dentro da própria família. O envolvimento de professores do ensino fundamental garantiu a possibilidade da repetição do assunto em trabalhos em sala de aula. Garcia-Zapata \& Marsden (1993) relataram sucesso com a inclusão de escolares em um programa de controle da transmissão vetorial em Mambaí, Goiás. A opção por atividades lúdicas para promoção de aprendizagem em saúde entre os escolares seguiu as evidências de autores que mostraram a efetividade dessa estratégia (Schall et al., 1993).

Outro alvo importante foi o profissional de saúde sendo estratégico na inserção de atividades a ele direcionadas, uma vez que a atual política sanitária no Brasil coloca os agentes comunitários de saúde (ACS) e os postos de saúde como a porta de acesso dos serviços de saúde à comunidade. Acrescenta-se a este fato que a participação desses profissionais nas expe- riências de educação popular, a partir dos anos 70 , trouxe para o setor saúde uma cultura de relação com as classes populares, apesar de ser um processo demorado e extremamente exigente de investimento profissional (Vasconcelos, 1999). O envolvimento de profissionais de postos de saúde como fonte de informação sobre doença de Chagas e controle de triatomíneos é fundamental e estratégico para sustentar as atividades descritas neste trabalho, uma vez que a FUNASA está em franco processo de desmantelamento.

Nos últimos trinta anos, a transmissão da doença de Chagas tem decrescido, não só no Brasil, como também numa grande parte das Américas, devendo-se atribuir ao uso de inseticidas contra os vetores, à melhoria das habitações, do padrão de vida e da educação (Dias, 1999). No entanto, existirá ainda um contingente de indivíduos já infectados cujo atendimento converte-se em questão básica para as autoridades sanitárias (Gontijo, 1976). Conseqüentemente, buscou-se esclarecer profissionais especialistas a fim de garantir melhor atendimento desde os bancos de sangue até o nível terciário de atenção médica.

A comunidade participou de forma ativa nas ações de vigilância e controle. Houve o envolvimento de acadêmicos na lógica de prevenção de agravos, contribuindo com a formação de profissionais compromissados com a inversão do modelo de saúde vigente no Brasil. Foi estabelecida uma relação de confiança profissional mútua entre universidade e técnicos / agentes da FUNASA, o que será de grande valia, antevendo o desmantelamento da FUNASA e a instabilidade dos funcionários e secretários de saúde dos municípios. Este trabalho foi uma experiência isolada envolvendo educação de diferentes extratos da comunidade, contudo é importante destacar que devem ser valorizadas todas as ações educativas que incentivem o desenvolvimento de autocuidado e responsabilidade na promoção da saúde, lembrando a necessidade de se realizar, posteriormente, pesquisa de avaliação da percepção das mensagens educativas do projeto.

\section{Agradecimentos}

Ao apoio financeiro do curso de pós-graduação em Ecologia de Ambientes Aquáticos Continentais, Universidade Estadual de Maringá (UEM) e Fundação Nacional de Saúde/UEM, convênio no 1899/98. 


\section{Referências}

ALEGRE, M. \& MORO, D. A., 1986. A mobilidade da população nas antigas áreas cafeeiras do Norte do Paraná. Boletim de Geografia (UEM), 4:28-73.

ALMEIDA, A. S., 1948. Doença de chagas no norte do Paraná. Anais Paulistas de Medicina Cirurgia, 56:29-36.

ARAÚJO, H. C. S., 1954. A doença de chagas no Paraná. Memórias do Instituto Oswaldo Cruz, 52: 477-485.

ARAÚJO, S. M.; ANDO, M. H.; CASSAROTTI, D. J.; MOTA, D. C. G. A.; BORGES, S. M. R. \& GOMES, M. L., 2000. Programa ACHEI: “Atenção ao chagásico com educação integral no Município de Maringá e região noroeste do Paraná", Brasil. Revista da Sociedade Brasileira de Medicina Tropical, 33: 565-572.

AVILA, M. G.; MARTINEZ, H. M.; PONCE, C.; PONCE, E. \& SOTO, H. R., 1998. Chagas disease in the central region of Honduras: Knowledge, beliefs and practices. Revista Panamericana de Salud Pública, 3:158-163.

CAMARGO, M. E.; SILVA, G. R.; CASTILHO, E. A. \& SILVEIRA, A. C., 1984. Inquérito sorológico da infecção chagásica no Brasil, 1975/1980. Revista do Instituto de Medicina Tropical de São Paulo, 26: 192-204.

DIAS, J. C. P., 1999. Problemas e possibilidades de participação comunitária no controle das grandes endemias. Cadernos de Saúde Pública,14:19-37.

DIAS, J. C. P. \& GARCIA, A. L. R., 1978. Vigilância epidemiologica com participacion comunitaria. Um programa de enfermedad de Chagas. Boletín de la Oficina Sanitaria Panamericana, 84:533-544.

FALAVIGNA-GUILHERME, A. L.; SILVA, S. V. \& ARAUJO, S. M., 1999. O Mal do Barbeiro. Maringá: Universidade Estadual do Maringá.

FALAVIGNA-GUILHERME, A. L.; PAVANELLI, G. C.; COSTA, A. L. \& ARAÚJO, S. M., 2001. Secondary triatomines species in dwellings and other nearby structures in municipalities under epidemiological surveillance in State of Paraná, Brazil. Revista Panamericana de Salud Pública, 9:385-392.

FALAVIGNA-GUILHERME, A. L.; PAVANELLI, G. C.; RODRIGUES, M.; SILVA, S. V. \& ARAÚJO, S. M., submetido. Infestação triatomínica e prevalência humana e de animais domésticos para o Trypanosoma cruzi em zona rural de municípios do Estado do Paraná, Brasil. Revista da Sociedade Brasileira de Medicina Tropical.

GARCIA-ZAPATA, M. T. A. \& MARSDEN, P. D., 1993. Chagas' disease: Control and surveillance through use of insecticides and community paticipation in Mambaí, Goiás, Brazil. Bulletin of the Pan American Health Organization, 27:265-279.

GONTIJO, E. D., 1976. Atendimento ao doador inapto por sorologia chagásica. Revista da Sociedade Brasileira de Medicina Tropical, 29:25-27.

HOLLANDA, H. H., 1992. Saúde como Compreensão de Vida. Um Manual de Educação para a Saúde. Brasília: Divisão Nacional de Educação Sanitária, Ministério da Saúde.

LOBO, A. G. S.; BORBA, A. M. \& SOUZA, J., 1952. Contribuição ao conhecimento da distribuição geográfica dos triatomíneos domiciliários e seus ín- dices de infecção natural pelo Schizotrypanum cruzi no Estado do Paraná. Revista Brasileira de Malariologia e Doenças Tropicais, 6:571-584.

MELLO, D. A.; PEDRAZZANI, E. S. \& PIZZAGATTI, C. P., 1992. Helmintoses intestinais: O processo de comunicação e informação no programa de educação e saúde em verminose. Cadernos de Saúde Pública, 8:77-82.

NASCIMENTO, C.; MARASSÁ, A. M.; CURADO, I. \& PIAZZA, R. M. F., 1997. Encontro de Panstrongylus megistus em ecótopo artificial: Domiciliação ou mera visitação. Revista da Sociedade Brasileira de Medicina Tropical, 30:333-336.

SCHALL, V. T.; DIAS, A. G. P.; MALAQUIAS, M. L. G. \& SANTOS, M. G., 1993. Educação em saúde em escolas públicas de 1o grau da periferia de Belo Horizonte, MG, Brasil. I Avaliação de um programa relativo à esquistossomose. Revista do Instituto de Medicina Tropical de São Paulo, 35:563-572.

SILVA, R. A.; BONIFÁCIO, P. R. \& WANDERLEY, D. M. V., 1999. Doença de Chagas no Estado de São Paulo: Comparação entre pesquisa ativa de triatomíneos em domicílios e notificação de sua presença pela população em área de vigilância entomológica. Revista da Sociedade Brasileira de Medicina Tropical, 32:653-659.

SILVEIRA, A. C.; FEITOSA, V. R. \& BORGES, R., 1984 Distribuição de triatomíneos capturados no ambiente domiciliar, no período 1975/83, Brasil. Revista Brasileira de Malariologia e Doenças Tropicais, 36:15-312.

SILVEIRA, A. C. \& RESENDE, D. F., 1994. Epidemiologia e controle da transmissão vetorial da doença de Chagas no Brasil. Revista da Sociedade Brasileira de Medicina Tropical, 27:11-22.

SILVEIRA, A. C. \& VINHAES, M., 1998. Doença de Chagas: Aspectos epidemiológicos e de controle. Revista da Sociedade Brasileira de Medicina Tropical, 31:15-60.

SIMÕES, A. J. P., 1943. Doença de chagas no Estado do Paraná, Brasil. Esboço epidemiológico. Memórias do Instituto Oswaldo Cruz, 39:279-290.

TOLEDO, M. J. O.; KÜHL, J. B.; SILVA, S. V.; GASPERI, M. V. \& ARAÚJO, S. M., 1997. Estudo sobre triatomíneos e reservatórios silvestres de Trypanosoma cruzi no Estado do Paraná, Sul do Brasil: Resultados preliminares. Revista da Sociedade Brasileira de Medicina Tropical, 30:197-203.

VASCONCELOS, E. M., 1999. Educação popular como instrumento de reorientação das estratégias de controle das doenças infecciosas e parasitárias. Cadernos de Saúde Pública, 14:39-57.

WILLIAMS-BANGLERO, S.; VANDBERG, J. L. \& TEIXEIRA, A. R., 1999. Attitudes towards Chagas'disease in an endemic Brazilian community. Cadernos de Saúde Pública, 15:7-13.

Recebido em 29 de novembro de 2000

Versão final reapresentada em 22 de maio de 2001 Aprovado em 19 de fevereiro de 2002 